Mer.-Differ. in

Zeit v. Par.aus

M. Z. Conjunction in mittlerer Zeit.

den Eintritten.

a) Berlin, Observalorium

b) Dresden, Wohnung des Hrn. Rasching

c) Göttingen, neue Sternwarte

d) Mannheim, Observatorium

e) Paris, Ecole militaire

f) Prag, Observatorium

g) Prag, Wohn. des Hrn. Hal laschka

h) Regensburg

i) Wien, Observatorium

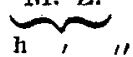

Eintritt $\begin{array}{lll}7 & 21 & 31,7\end{array}$

Eintritt 723 1,0

Austritt

Eintritt

Austritt

Eintritt

Eintritt

Eintrilt

Eintritt

Austritt

Eintritt

Austritt

Eintritt

Austritt
8462,0

720,46

$\begin{array}{lll}8 & 25 & 2,2\end{array}$

$\begin{array}{lll}6 & 52 & 35,45\end{array}$

$\begin{array}{lll}6 & 17 & 4,19\end{array}$

$\begin{array}{lll}7 & 26 & 39,8\end{array}$

$\begin{array}{lll}726 & 40,5\end{array}$

$8 \quad 4952,0$

$\begin{array}{lll}7 & 13 & 19,0\end{array}$

$\begin{array}{llll}8 & 37 & 39,0\end{array}$

$\begin{array}{lll}7 & 38 & 8,2\end{array}$

9043,8 h, , "

$7^{h}{ }^{\prime}{ }^{\prime}{ }^{\prime \prime} I^{\prime \prime}, 52+2,0823 \Delta R+0,4413 \Delta B-0,7542 \Delta \pi$

$72319,27+2,0518 \Delta R+0,2616 \triangle B-0,6711 \Delta \pi$

$72344,78-2,0522 \Delta R+0,2657 \Delta B-1,0816 \Delta \pi$

$787,11+2,0756 \Delta R+0,4084 \Delta B-0,6301 \Delta \pi$

$7818,91-2,0435 \Delta R+0,1867 \Delta B-0,9422 \Delta \pi$

$7_{2} 21,55+2,0450 \triangle R+0,2172 \Delta B-0,4782 \Delta \pi$

$63733,90+2,0648 \Delta R+0,3493 \triangle B-0,3332 \triangle \pi$

$7261,36+2,0395 \Delta R+0,1358 \Delta B-0,6331 \Delta \pi$

$7261,83+2,0395 \Delta \mathrm{R}+0,1358 \Delta \mathrm{B}-0,6332 \Delta \pi$

$72623,16-2,0710 \Delta R+0,3849 \Delta B-1,1560 \Delta \pi$

$71644,86+2,0360 \Delta R+0,045 \Delta B-0,5270 \Delta \pi$

7 I7 14,16-2,0989 $\triangle R+0,5141 \triangle B-1,1523 \triangle \pi$

$73353,20+2,0390 \Delta R-0,128 z \Delta B-0,5891 \triangle \pi$

7 $3414,77-2,1305 \Delta R+0,6310 \Delta B-1,3148 \Delta \pi$

$$
\begin{array}{r}
\underbrace{\prime \prime}_{1 \prime \prime} \\
-4410,0 \\
-4537,8 \\
-3025,6 \\
-2430,1 \\
+\quad 7,6) \\
-4819,9 \\
-4820,3 \\
-393,4 \\
-5611,7
\end{array}
$$

S.

\title{
Beobachtungen in New South Wales.
}

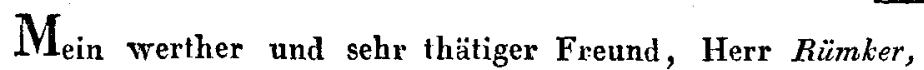
hat kaum ein Jahr Europa verlassen, und schon habe ich das Vergnügen, das von ihm mit einem Reichenbach'schen Wiederhohlungskreise in Paramatta beobachtete Winter8olstiz von 1821, als die erste Frucht der dort entstehenden Sternwarte, vorlegen zu können. Dies, und die andern hier bekannt gemachten Beobachtungen, sind aus einem Briefe an mich, und zwei mir gütigst mitgetheilten Briefen an Herrn Doctor Olbers gezogen, die er gegen Ende Januars in Australien geschrieben hat.

Der Ban der Sternwarte in Paramatta rückt rasch fort. Bis zu ihrer Vollendung ist ein Reichentach'scher Kreis in einem nahen Hzuse, das unter derselben Breite liegt, aufgestellt.

Aus den bisher mit diesem Wiederhohlungskreise gemachten Sternbeobachtungen folgt die südliche Breite dicses Hauses $33^{\circ} 48^{\prime} 46^{\prime \prime}, 7$

Zur vorläufigen Bestimmung der Länge von Paramatta sind folgende Beobachtungen gemacht.

\section{Verfinsterungen von Jupiterstrabanten.}

Tag. $\underbrace{\text { Trab. }}$

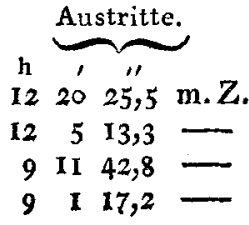

Länge.

1821 Dec. 8

Dec. 14 II

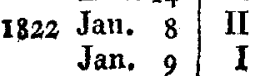

\begin{tabular}{l|l|rrr} 
Jan. 9 & 1 & 9 & 1 & 17,2
\end{tabular}

Mittel
Herr Rïmker fiihrt nicht an, nach welchen Tafeln er die Länge daraus berechnet hat, eben so wenig von wo an er sie zählt, die letzte Auslassung ist leicht zu suppliren, da sie offenbar von Greenwich gerechnet ist.

\section{Mondsdistanzen.}

Jede Distanz ist ein Mittel von 4 oder mehreren mit verschiedenen Instrumenten beobachteten. Die Hölien sind mit der wahren Zeit und mit dem Winkel des Radius mit dem Aequator $=33^{\circ} 39^{\prime}$ anstatt der Breite berechnet.

$$
\text { Weitin scheinb. Ab- }
$$

w. Zeit in scheinb. scheinb. stand des Länge von

1822. Paramatta. Ohöhe. (Chöhe. Mittelpuncts. Greenw.

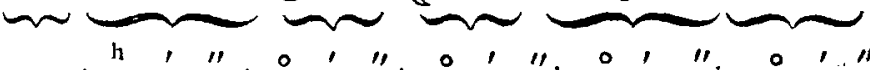

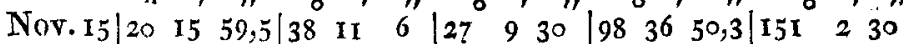
\begin{tabular}{lllll|llll|llll|lll}
20 & 23 & 17,5 & 39 & 42 & 0 & 26 & 10 & 6 & 98 & 34 & 18,9 & 151 & 5 & 15
\end{tabular}

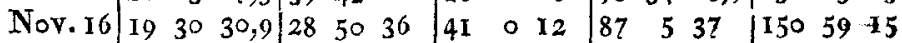
\begin{tabular}{lll|llll|llll|lll|llll}
19 & 34 & 49,7 & 29 & 45 & 2 & 40 & 41 & 58 & 87 & 4 & 35 & 151 & 6 & 10
\end{tabular}

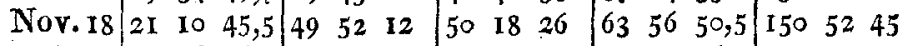
$\begin{array}{llllllllllllllllll}21 & 16 & 16,5 & 50 & 59 & 40 & 49 & 41 & 29 & 63 & 55 & 36,2 & 151 & 4 & 7\end{array}$ \begin{tabular}{lll|lll|llll|lllll}
21 & 43 & 27 & 56 & 49 & 2 & 47 & 35 & 54 & 63 & 47 & 20,5 & 150 & 47 & 45
\end{tabular}

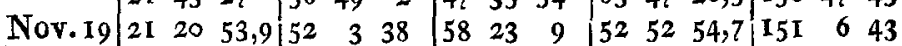

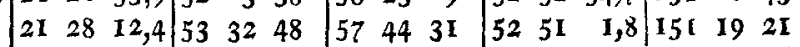
\begin{tabular}{llllll|llll|llllllll}
21 & 35 & 32,4 & 55 & 1 & 0 & 57 & 4 & 28 & 52 & 48 & 51,0 & 150 & 51 & 36
\end{tabular} $\begin{array}{llllllllllllllll}21 & 47 & 39,8 & 57 & 28 & 0 & 55 & 41 & 5 & 52 & 45 & 22,5 & \text { I } 5 \text { I } & \circ & 5 \text { I }\end{array}$ \begin{tabular}{lll|lll|lll|lll|lll}
21 & 51 & 47 & 58 & 16 & 0 & 55 & 16 & 47 & 52 & 44 & 11 & 150 & 45 & 0
\end{tabular} $\begin{array}{llllllllllllllll}21 & 56 & 41,4 & 59 & 14 & 30 & 54 & 39 & \text { I8 } & 52 & 43 & 12 & 151 & 15 & 21\end{array}$

Mittel 15 I 110

oder in Zeit xoh $4^{\prime} 5^{\prime \prime}$ von Greenwich. 
Durch 7 Chronomeler ist die Länge des Gouvernemenlshauses in Sidney $=12^{\prime} 38^{\prime \prime}, 1$ in Bugen von Paramatta bestimmt, mithin ist die Länge des Gouvernementshauses in Sidney

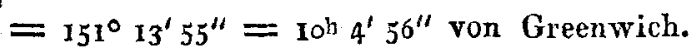

Die eben angeführten 7 Chronometer haben die Länge von Paramatla $=10^{h} 6^{\prime} 31^{\prime \prime}$ ibertragen. Begreiflicherweise aber steht dies Resultat an (jewilsheit den Jupiterstrabanten, and Mondsdistanzen nach.

\section{Sternbedeckungen.}

3821 Dec. I4 $\rho$ Leonis Fintr. $14^{\text {h }} 47^{\prime} 9^{\prime \prime}, 1 \mathrm{~m}$. Z. in Param. Ausir. 154557

Fs ist nicht ganz gewifs, ob der Austritt von demselben, oder von einem andern Sterne beobachtet ist. Doch ist es wahrscheinlich auch $\rho$ Leonis.

1821 Dec. 297.8 Gröfse Eintrilt $8^{\mathrm{h}} 33^{\prime} 47^{\prime \prime} \mathrm{m}$. $\mathrm{Z}$.

$$
\text { 7. } 8 \text { Gröfse Eintrilt } 8 \quad 4647
$$

1822 Jan. I6 7Gröfse 60 Eintritt $16 \quad 8 \quad 6,9-$ 6.7 Gröfse 69 Eintritt $16 \quad 54 \quad 99,9-$

Nachdem diese Bestimmungen der Länge und Breite der Sternwarte in Paramatta vorangeschickt sind, wollen wir die Beobachtungen des Wintersolstizes 1821 folgen lassen.

Wintersolstiz 1821 mit Reichenbactss Kreis beobachtet. WahreZen.-Dist.

d. Mittelpuncts Gorr.weg. Reduct.auf Zenithdistanz

1821 derSonne. derBreite. das Solstiz.

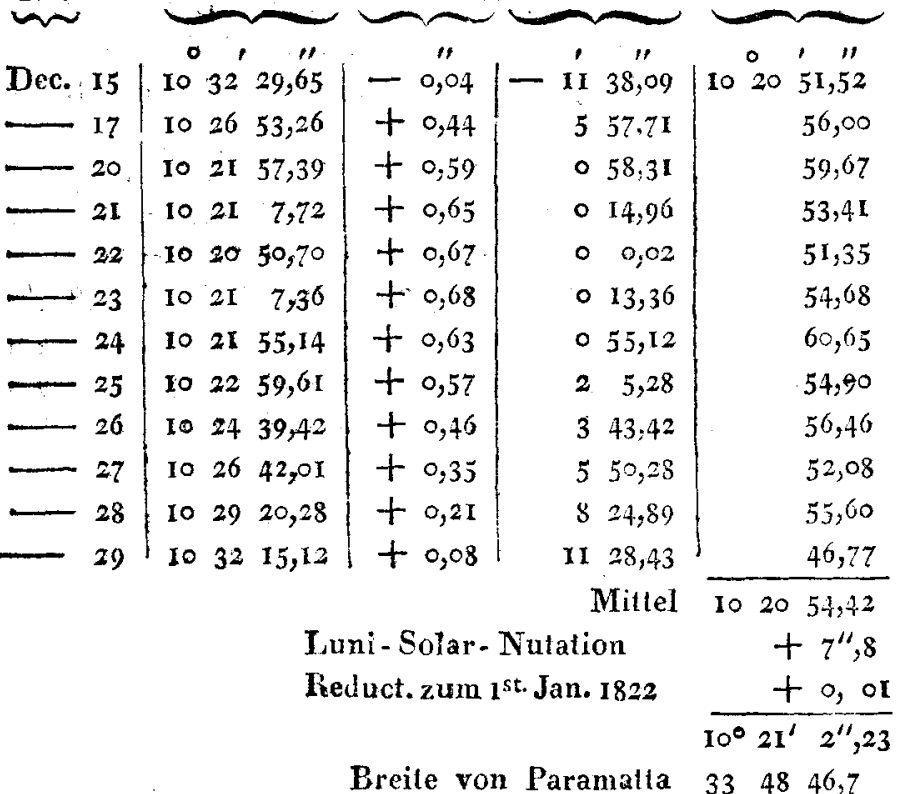

\begin{tabular}{rrrr} 
Breite von Paramatia & $33 \quad 48$ & 46,7 \\
\hline Mittlere Schiefe d. Ecliptic Jan. 1. 1822 & $23^{\circ} \quad 27^{\prime} 44^{\prime \prime}, 5$
\end{tabular}
Die Correction wegen der Sonnenbreite ist nach der Formel $d \omega=\lambda \cdot \frac{\cos \omega}{\cos D}$ gerechnet. Bei den Berechnungen selbst hat Herr Rïmker mehr Decimalen gelraucht, als hier angegeben sind. Er hat auch weilläuftig die dabei gebrauchten Methoden entwickelt, auf die ich es mir vorbebalte, ein andermal zurückzukommen.

\section{Matsnetisehe Beobachtungen in Paramatta.}

1821 Nov. 12 ward die Inclination an Gambey's Instrument abgelesen.

$$
62^{\circ} 36^{\prime} 19^{\prime \prime}
$$

Die Zahl der Schwingungen in $5^{\prime}$ war

$$
\begin{aligned}
& \text { im magnetischen Meridian }=128 \\
& \text { im magnet. ersten Vertical }=120,8
\end{aligned}
$$

also $\left(\frac{T}{T^{\prime \prime}}\right)^{2}=\cos 62^{\circ} 57^{\prime}$

Ausser diesen in Paramatta gemachten Beobachtungen enthalten die Briefe noch mehrere auf der Reise selbst gemachte Ortsbestimmungen, Peilungen und magnetische

\begin{tabular}{|c|c|c|c|c|c|}
\hline$\$ 1821$. & B & $\mathbf{L}$. & c. & S.d.C. & Abweichung \\
\hline Aug. 24 & $23^{\circ} 27^{\prime} \mathrm{S}$ & $42^{\circ} 57^{\prime} \mathrm{W}$ & s.s.o. & s.v. & $2^{\circ} 12^{\circ} 0$. \\
\hline 25 & 2540 & 4148 & S.z. O. & s. v. & 242 \\
\hline 26 & 2745 & 404 & 5.0, z. O & l. h. & 229 \\
\hline 27 & 2916 & 3744 & 0.3 .0 & 1. h. & 21 \\
\hline 28 & 3021 & 3533 & o.s.o. & s.h. & $\circ 55$ \\
\hline Sepl. 0 & $37 \quad 18$ & 1025 & O. 5.0 & 1. h. & $1546 \mathrm{WW}$. \\
\hline$?$ & $38 \quad 8$ & 74 & O.z.S. & l. h. & I4 3 \\
\hline 10 & 357 & $\circ 30$. & N.O.z.O. & s. v. & 2043 \\
\hline 13 & 392 & 616 & 5.0.z.o. & l. h. & 2323 \\
\hline 15 & 3920 & I2 7 & S.O.z.o. & s. v. & $26 \quad 49$ \\
\hline 16 & $393^{6}$ & 1453 & S.O.z.O. & s. v. & 2612 \\
\hline 17 & $393^{6}$ & $17 \cdot 2$ & S.O.z.O. & s. h. & 2742 \\
\hline 18 & 30.42 & 203 & S.0.z.o. & $\left\{\begin{array}{l}1 . h . \\
\text { s.h. }\end{array}\right.$ & $\begin{array}{ll}27 & 58 \\
28 & 37\end{array}$ \\
\hline 24 & $38 \quad 45$ & 2749 & s. 0 . & s. s. & 3154 \\
\hline 27 & 3634 & $36 \quad 4$ & N. O. $\frac{11}{2} \mathrm{O}$ & s. v. & 3029 \\
\hline 28 & $36 \quad 59$ & 3624 & S.O. $\frac{\pi}{2} \mathrm{~S}$. & 1. h. & 297 \\
\hline Octbr. 3 & 3735 & $5 I 3 I$ & S.O.z.S. & 1. h. & 3132 \\
\hline 4 & $38 \quad 23$ & $543 x$ & S.0.z.0. & 1. h. & 2529 \\
\hline 0 & 3929 & 6240 & S.0.z.0. & 1. h. & 2520 \\
\hline II & $384 \mathrm{I}$ & 7739 & O. z.N. & l. h. & 18 xo \\
\hline
\end{tabular}
Beobachtungen, die ich hier folgen lasse. Die Buchstaben der Veberschrift haben diesellje Bedeutung wie bei Rümkers früheren Beobachtungen.

Magnetische Beobachtungen auf der Reise. 
1821.

Octbr. 12

\begin{tabular}{|c|c|c|c|}
\hline tod & 12 & (bey St & Pauls) \\
\hline & 14 & $38^{\circ}{ }_{5} \mathrm{I}^{\prime} \mathrm{S}$. & $85^{\circ} 49^{\prime} \mathrm{O}$ \\
\hline & 17 & 392 & 9515 \\
\hline & 18 & 3918 & 9828 \\
\hline & 20 & $\begin{array}{ll}39 & 24\end{array}$ & 1073 \\
\hline & 21 & 395 & $\begin{array}{lll}113 & 13\end{array}$ \\
\hline & 22 & 3840 & 11435 \\
\hline & 23 & $38 \quad 27$ & 11720 \\
\hline & 27 & $395 \mathrm{I}$ & 12358 \\
\hline & 28 & 3937 & $127 \quad 54$ \\
\hline & 31 & $38 \quad 39$ & 1386 \\
\hline Nov. & 3 & 3934 & $148 \quad 15$ \\
\hline & 5 & 3539 & 15152 \\
\hline & 6 & 353 & $151 \quad 12$ \\
\hline
\end{tabular}

C S.d.C. Abweichung

W.z.S. | 1. h. $\mid 22^{\circ} 35^{\prime} \mathrm{W}$. O.S.O. 1.h. I8 52 O.S.O. s.h. 1840 O.S.O. s.h. 1557 O.z.S. \{l.h. I4 10 O.z. S. 1. h. O. $\frac{\mathrm{I}}{2} \mathrm{~S}$. 1. h. 1233 * O. s. v. 945 O. $\frac{\pi}{2}$ S. 1. s. $85 \mathrm{I}$ O. $\frac{1}{2}$ N. 1.h. 337 O.z.N. s. v. 40 o. 1. h. 3 i 0 . N. O. 1.h. 859 N. N.W. s. s. io 54 W.N.W.I 1.h. $9273^{\circ}$ Inclinationen der Magnetnadel.

1821.

L. ,

C.

S. d. I. Inclination

\begin{tabular}{|c|c|c|c|c|c|}
\hline Aug. 27 & $29^{\circ} 18^{\prime} \mathrm{S}$. & $37^{\prime} 36^{\prime} \mathrm{W}$ & O.S. 0 & s. h. & $30^{\circ} 33^{\prime}$ \\
\hline 28 & 3021 & 3534 & O.S.O. & s. h. & 3110 \\
\hline Sept. 4 & 349 & 1558 & O.S. O. & s. h. & 3358 \\
\hline 8 & 38 II & 314 & O. & s. h. & 4745 \\
\hline 9 & 3641 & 026 & N.O. & s. h. & $42 I$ \\
\hline 10 & 358 & $\circ \quad 140$ & S.S.W. & s. h. & $45 \quad 14$ \\
\hline II & 3655 & I I & S. S. o. & s. h. & 487 \\
\hline 13 & $\begin{array}{ll}39 & 3\end{array}$ & 623 & S.O.z.S. & s. h. & $\begin{array}{ll}52 & 44\end{array}$ \\
\hline 16 & 3938 & 1457 & O. S.O. & s. h. & 556 \\
\hline 17 & $393^{6}$ & 175 & S.O.z.O & s. h. & $56 \quad 58$ \\
\hline 18 & 3945 & 2017 & S.O.z.O. & s. h. & $58 \quad 23$ \\
\hline 19 & 3955 & 2218 & O.z.S. & s. h. & $57 \quad 24$ \\
\hline 23 & 3826 & .2548 & S.O.z.O. & s. h. & 5941 \\
\hline 24 & $38 \quad 54$ & 2825 & S. O. & s. h. & 6052 \\
\hline 27 & 3650 & $3^{6} 7$ & N.O.z.O. & s. h. & 6131 \\
\hline Octbr. 4 & $38 \quad 29$ & 5433 & S.O.z.O. & s. h. & 6527 \\
\hline 12 & (Bey St. & Pauls) & W.z.N. & s. h. & $66 \quad 19$ \\
\hline 16 & 395 & 9324 & 0. S. 0 . & s. h. & 6745 \\
\hline 17 & 397 & 9529 & O. S. 0 . & s. h. & 6918 \\
\hline 19 & $39=7$ & 10311 & S. $\frac{1}{2} O$. & s. h. & 6845 \\
\hline 22 & $38 \quad 46$ & 11357 & 0 & s. h. & $68 \quad 49$ \\
\hline 26 & $385^{\circ}$ & I $2 \mathrm{I} 9$ & S.O.z.O. & s. h. & 6831 \\
\hline Nov. 12 & in Paran & alta & & & 6236 \\
\hline
\end{tabular}

Ortsbestimmungen.

Lage von St. Pauls.

Am 12ten October wurde der Crater des Vulcans auf St. Pauls, die südlichere Insel der beiden, (dio mit der nörllichern, Amsterdam, häufig verwechselt wird) in SW.z.W. $\frac{7}{2}$ W.pr. Compals gepeilt in der Entfernung von $2^{\prime}$; die beobachtele Breite damit reuucirt, giebt Breite des Craters $38^{\circ} 44^{\prime} 36^{\prime \prime} \mathrm{S}$.

\section{Länge derselben durch}

Mondsabstände $77^{\circ} 33^{\prime} 18^{\prime \prime}$ Ost ron Greenwich.

Chronometer $\quad 78 \quad 7 \quad 28$

Es war hier hohes Wasser ohngefähr um 2 Uhr Nachmillas; Fahrenheits Thermometer stieg auf $184^{\circ}$ in den aus den Steinen hervorsprudelnden heifsen Quellen, die zur Zeit des hohen Wassers mit See bedeckt sind; im Sande nebenan stand es auf $150^{\circ}$.

\section{Lage der Albion-Insel.}

Diese von Capitain Turnbull gesehene, und ron ihm Albion genannte Insel passirten wir zur Nachtzeit; der $3^{\text {te }}$ Steuermann und verschiedene der Mannschaft versichern sie deutlich gesehen, und in N.z.O. $\frac{1}{2}$ O. $16^{\prime}$ gepeilt zu haben: daraus wïrde die folgende Lage folgen:

Breite $38^{\circ} 39^{\prime}$ S. Länge $\left\{\begin{array}{l}\text { Mondsabst. } 139^{\circ} 55^{\prime} \text { O. v. Greenw. } \\ \text { Chronom. 140 19 }\end{array}\right.$ Abweichung der Magnetnadel $3^{\circ}$ östlich.

\section{Lage verschiedener Punkte in Bals-Strafse.}

Am $3^{\text {ten }}$ November passirle das Schiff durch die BarsStrafse, und hatte ron Tages-Anbruch bis Nacht eine ziemlich gleichförmige Fahrt von 7 Mexlen, aber dabei anfangs mit der Fluth zu kämpfen. - Während dieser Zeit wurden von Zeit zu Zeit Sonnenhöhen an dem Chronometer von Bréguet beobachtet, und das Land mit dem. Schiffs-Compals gepeilt; hernach beobachtete Sir Thomas Brisbane und Herr Rïmker mit verschiedenen Instrumenten, melirere Abstänle des Mondes von der Sonne, Venus und Jupiter, woraus die Längen der folgenden Tafel hergeleitet sind. 


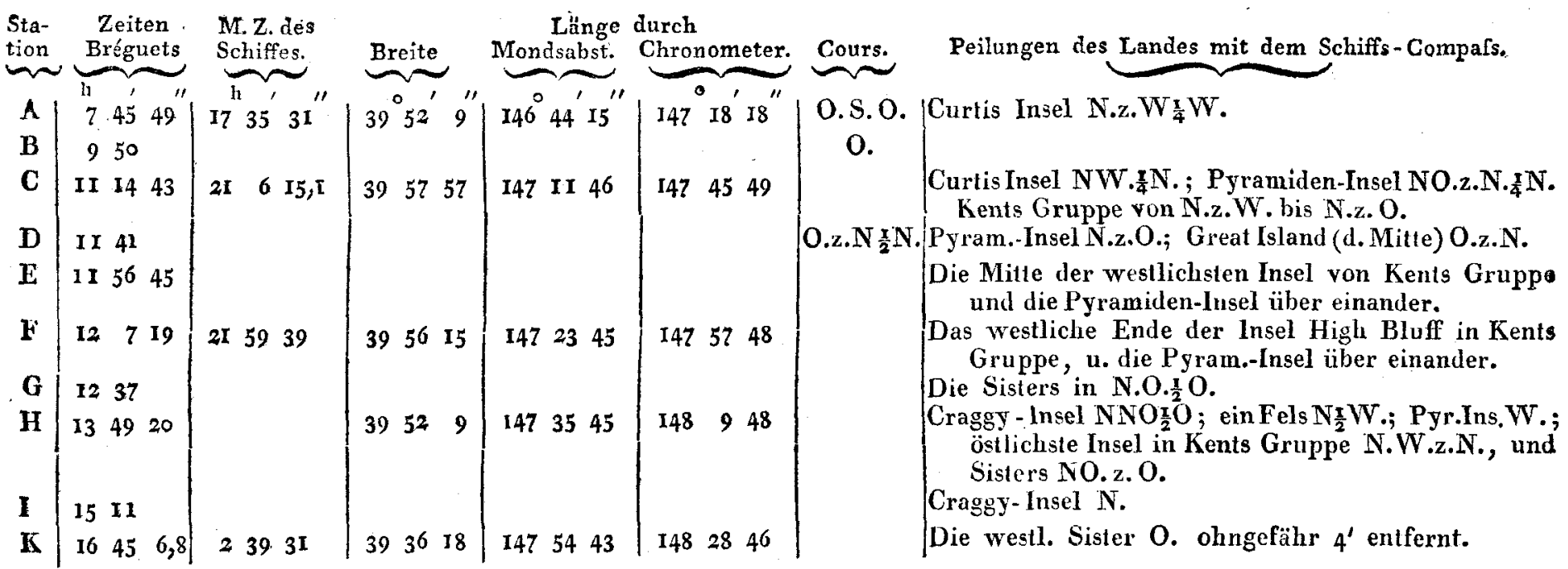

Hieraus, und durch Hülfe einer Charte von Flinders oder Freycinet, können Hydrographen die geographische Lage dieser Inseln ableiten.

\section{Ch. Rümker.}

\section{A $n \quad z \quad$ e $\quad$ i $g$ e.}

$D_{a}$ mit dem 24rten Stüicke der erste Band dieser A stro nomischen Nachrichten geschlossen, und nach dessen Schlusse, die Frei-Exemplare ausgenommen, kein Exemplar der Fortsetzung mehr olıne Voranshezahlung versandt wird, so ersuche ich alle Herren Subscribenten bei Zeiten deswegen die Bestellungen bei mir zu machen, und die Gelder einzusenden, oder anzuweisen. Der Preis eines Bandes von 24 Bogen ist hier 8 me Courant, oder ein holländischer Ducaten. Wer seine Exemplare durch ein Postamt, oder durch den Buchhandel zieht, mufs nach Verhältnifs mehr bezahlen. Um diesen Weg zu erleichtern, will ieh den Post-Aemtern und Buchhandlungen bei ihren Bestellungen Rabalt geben, wogrgen sie, wie alle andere Subscribenten, jemand hier, oder in Hamburg zu nennen haben, dem die Exemplare, so wie sie erscheinen, übergeben werden.

Schumacher (Altona Palmaille 441.)

\section{$I$ in $h$ a 1 t.}

Chronometrische Bestimmung des Längenunterschiedes zwischen Copenhagen und Hamburg. (Beschlufs.) pag. 305. Aus einem Schreiben des Herm Dr. Olbers in Bremen an den Herausgeber. pag. 307.

Auszug aus einem Briefe des Herrn Dr. Olbers an den Herausgeber. pag. 307.

Elemente des ersten Cometen im Fuhrmann. pag. 309.

Berechnung der Sternbecieckung 1 A Tauri 1818 Febr. 13 von Herrn Vietż in Lübeck. pag. 311.

Rümker Beobachtungen in New South Wales. pag. 313.

Anzeige. pag. 320 .

Allona im September 1822. (Hiebei ein Blatt Druckfehler.) 